\title{
An unusual case of septate uterus with double cervix and longitudinal vaginal septum simulating uterus didelphys
}

\author{
Alka Vijay*, Archana Salve, Kshitij Murdia, Vipin Chandra
}

Indira IVF private limited, Udaipur, Rajasthan, India

Received: 20 October 2016

Revised: 21 October 2016

Accepted: 15 November 2016

\author{
*Correspondence: \\ Dr. Alka Vijay, \\ E-mail: alkajiya28@gmail.com
}

Copyright: () the author(s), publisher and licensee Medip Academy. This is an open-access article distributed under the terms of the Creative Commons Attribution Non-Commercial License, which permits unrestricted non-commercial use, distribution, and reproduction in any medium, provided the original work is properly cited.

\begin{abstract}
Rare mullerian anomalies not falling in any present classification are sometimes reported. A 27-year-old woman came to our hospital with history of secondary infertility. She was found to have a longitudinal vaginal septum, cervical duplication and two endometrial cavities, separated by a complete septum. Laparoscopic examination revealed a relatively intact uterine fundus with both patent fallopian tubes. Hysteroscopic exam confirmed the presence of double vagina and double cervix, as well as complete uterine septum. It was a rare variant of complete septate uterus with double cervix, which could be successfully treated by hysteroscopic operation. Diagnosis and management of this unusual Müllerian anomaly are discussed in the context of a literature review.
\end{abstract}

Keywords: Double cervix, Hysteroscopic septotomy, Longitudinal vaginal septum, Septate uterus

\section{INTRODUCTION}

The classification of uterine anomalies divides the septate uterus (class V, American fertility society) into partial (sub- septate) or complete septate groups if the septum approaches the internal os. ${ }^{1}$ A rare variant of the septate uterus, as combined with cervical duplication and a longitudinal vaginal septum, has been anecdotally reported. ${ }^{2,3}$ The unidirectional caudal to a cephalic fusion of the Müllerian ducts is incompatible with the presence of two cervices and a single unified uterine fundus and reinforces the alternative bidirectional theory. ${ }^{4,5} \mathrm{We}$ report the case of a woman with this rare anomaly.

\section{CASE REPORT}

A 27-year-old lady came to our hospital with a 4-year history of secondary infertility and suspicious uterovaginal anomaly. She had regular menstrual cycles ranged 25 to 30 days with normal flow lasting for 4-5 days with no complaints of dysmenorrhea. She did not have any difficulty in intercourse. She had undergone diagnostic laparoscopy at some other center and was told that she had double the uterus. The patient underwent three cycles of IUI and 1cycle of IVF. She conceived in IUI cycle but had missed abortion at around seven weeks after that patient came to our hospital. Pelvic examination revealed a longitudinal vaginal septum with a more pliable right vagina and comparatively smaller left vaginal canal. Two separate cervices were clearly visible (Figure 1).

3D ultrasonography showed uterus with a minimal indentation at fundus along with two endometrial cavities and two well-developed cervices (Figure 2). An abdominal ultrasound showed the presence of two kidneys.

We decided to perform hysteroscopy under transabdominal USG guidance for the evaluation and correction of the uterovaginal anomaly. Firstly a diagnostic hysteroscopy was performed. One12-French Foley catheter was inserted into the uterus through the right cervix and inflated with $4 \mathrm{ml}$ normal saline. Hysteroscope gently inserted into the left uterine cavity. A bulge was seen in the left uterine cavity and a small rent created using $5 \mathrm{Fr}$ scissor. Now hysteroscope introduced in the right cavity and rent enlarged. 
A communication established between two cavities just above internal os. Final hysteroscopic septotomy performed under the guidance of transabdominal sonography, and the entire septum resected using hysteroscopic scissors. Finally, a single roomy cavity achieved (Figure 3).

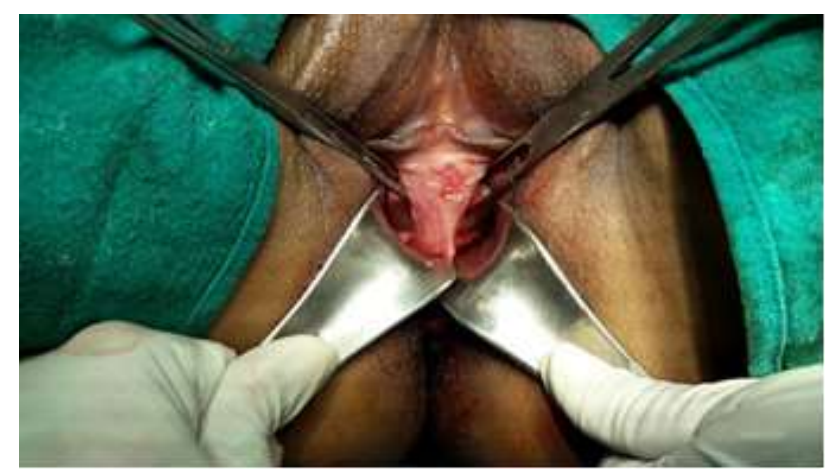

Figure 1: Per speculum examination showing longitudinal vaginal septum and two cervices.

Unification of the cervix was not done to avoid cervical incompetence later on. We did not excise the longitudinal vaginal septum, as she did not have any difficulty in sexual intercourse. Her postoperative course was unremarkable, and she was discharged one day later without any events. We recommended oral cyclic estrogen-progestin for three months. After treatment, she is now trying to go for pregnancy by ovarian stimulation.

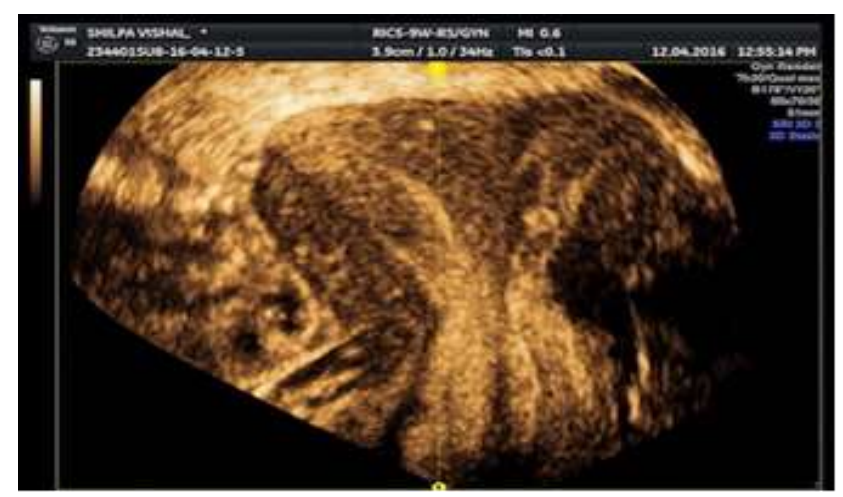

Figure 2: 3D USG showing two endometrial cavities and cervices.

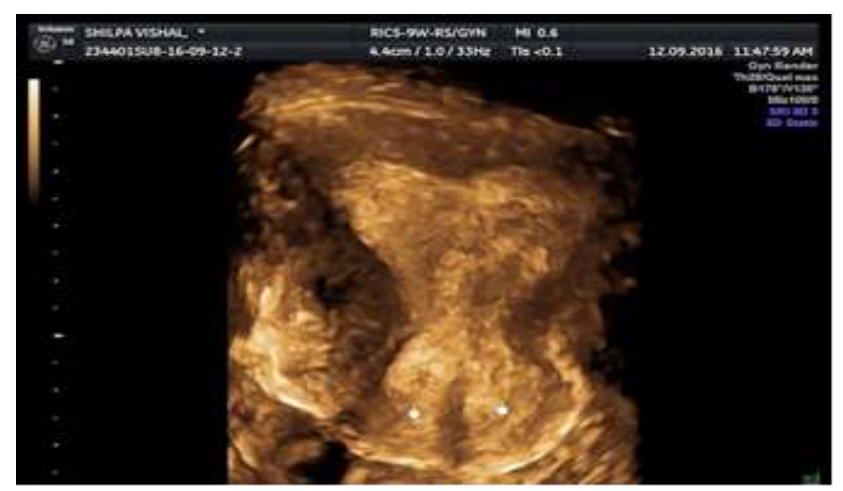

Figure 3: Post-operative 3D USG showing unification of two endometrial cavities.

\section{DISCUSSION}

Septate uterus with cervical duplication and a longitudinal vaginal septum is a rare congenital malformation. The most common misdiagnosis was the didelphic uterus. The unique characteristic of this malformation, a cervical duplication with a complete vaginal septum with a unified uterus, even in the presence of a complete septum, indicates that fusion initiated during development in the central portion of Müllerian ducts and extended cranially and caudally, as proposed by Musset et al. in $1967 .^{6}$ Location of fusion initiation may vary among individuals, and this variability could explain the wide variety of uterine malformations observed.

Patients described in the literature have presented with a variety of symptoms, including severe dysmenorrhea and infertility; some have been asymptomatic. Interestingly, a vast majority of patients, such as the present one, did not have difficulty with the initiation of intercourse. Diagnosis is made by careful examination, followed by imaging. Most physicians, however, still use a combined approach with hysteroscopy and laparoscopy to confirm a diagnosis.

The best approach to management, which should provide relief of symptoms and preserve reproductive ability, is controversial. Resection of the vaginal septum is easy and commonly performed. Hysteroscopic resection of a uterine septum using a minimally invasive approach (improving obstetric outcomes) is the gold standard according to most authorities. ${ }^{7-10}$ There have been different methods reported to distend or indent the septum for the hysteroscopic incision, including the use of metal probes, Foley catheters, or plastic dilators. The union of the two cervices is another area of controversy. Some authors believe that cervical manipulation increases the risk of cervical incompetence and that there is a risk of problematic bleeding during surgery. ${ }^{7-10}$ Others prefer to unify the cervices to facilitate surgery and decrease the likelihood of recurrent symptoms.

Funding: No funding sources

Conflict of interest: None declared

Ethical approval: Not required

\section{REFERENCES}

1. The American Fertility Society. The American Fertility Society classi- fications of adnexal adhesions, distal tubal occlusion, tubal occlusion secondary to tubal ligation, tubal pregnancies, Müllerian anomalies and intrauterine adhesions. Fertil Steril. 1988;49:944-55.

2. Pavone ME, King JA, Vlahos N. Septate uterus with cervical duplication and a longitudinal vaginal septum: a mullerian anomaly without a classification. Fertil Steril. 2006;85:494.e9-10. 
3. Acien P, Acien M, Sanchez-Ferrer ML. Mullerian anomalies "without a classification": from the didelphys-unicollis uterus to the bicervical uterus with or without septate vagina. Fertil Steril. 2009;91:2369.

4. Taylor E, Gomel V. The uterus and fertility. Fertil Steril. 2008;89:1-16.

5. Wang JH, Xu KH, Lin J, Chen XZ. Hysteroscopic septum resection of complete septate uterus with cervical duplication, sparing the double cervix in patients with recurrent spontaneous abortions or infertility. Fertil Steril. 2009;91:2643-9.

6. Musset R, Muller P, Netter A, Solal R, Vinourd JC, Gillet JY. Etat du haut appareil urinaire chez les porteuses de malformations uterines. Etude de 133 observations. Presse Med. 1967;75(26):1227-32.

7. Patton PE, Novy MJ, Lee DM, Hickok LR. The diagnosis and reproductive outcome after surgical treatment of the complete septate uterus, duplicated cervix and vaginal septum. Am J Obstet Gynecol. 2004;190:1669-75.

8. Giraldo JL, Habana A, Duleba AJ, Dokras A. Septate uterus associated with cervical duplication and vaginal septum. J Am Assoc Gynecol Laparosc. 2000;7(2):277-9.

9. Balasch J, Moreno E, Martinez-Román S, Moliní JL, Torné A, Sánchez-Martín F, et al. Septate uterus with cervical duplication and longitudinal vaginal septum: a report of three new cases. Eur J Obstet Gynecol Reprod Biol. 1996;65(2):241-3.

10. Sharara FI. Complete uterine septum with cervical duplication, longitudinal vaginal septum and duplication of a renal collecting system. A case report. J Reprod Med. 1998;43(12):1055-9.

Cite this article as: Vijay A, Salve A, Murdia K, Chandra V. An unusual case of septate uterus with double cervix and longitudinal vaginal septum simulating uterus didelphys. Int J Reprod Contracept Obstet Gynecol 2017;6:303-5. 